\title{
Noninvasive markers for esophageal varices in children with cirrhosis
}

\author{
Parisa Rahmani, MD¹, Fatemeh Farahmand, MD¹, Ghobad Heidari, MD², Azadeh Sayarifard, MD ${ }^{3}$ \\ ${ }^{1}$ Pediatric Gastroenterology and Hepatology Research Center, Tehran University of Medical Sciences, Tehran, Iran; ${ }^{2}$ Department of Pediatrics, Faculty of Medicine, \\ Lorestan University of Medical Sciences, Khorramabad; ${ }^{3}$ Growth and Development Research Center, Tehran University of Medical Sciences, Tehran, Iran
}

\begin{abstract}
Background: The diagnosis of esophageal varices (EV) is based on the findings of esophagogastroduodenoscopy (EGD), biopsy, and serum markers. Thus, noninvasive cost-effective tests through which high-risk EV children can be diagnosed are needed.
\end{abstract}

Purpose: This cross-sectional study aimed to identify the noninvasive markers for $\mathrm{EV}$ in children with liver cirrhosis.

Methods: A total of 98 children with liver cirrhosis were evaluated in this study. The spleen size, platelet count, serum albumin, liver function test results, and risk scores were evaluated prior to endoscopy. The endoscopic investigations aimed to identify the presence of $\mathrm{EV}$ and red signs, and determine varices sizes.

Results: Endoscopy revealed varices in 43 subjects (43.9\%). The spleen size, platelet count, international normalized ratio, aspartate aminotransferase to platelet ratio index (APRI), platelet count to spleen size ratio, and risk score differed significantly between patients with and without EV on univariate analysis; however, the logistic regression analysis showed no differences, indicating that none of these parameters were independently associated with the presence of EV.

Conclusion: Platelet count, risk score, platelet count to spleen size, and APRI can be useful tools for the identification of highrisk patients with $\mathrm{EV}$ and might reduce the need for invasive methods like EGD.

Key words: Portal hypertension, Gastroesophageal varices, Liver cirrhosis, Pediatrics, Gastrointestinal endoscopy

\section{Key message}

Question: Can noninvasive biomarkers identify esophageal varices among children with esophageal cirrhosis?

Finding: The spleen size, platelet count, international normalized ratio, aspartate aminotransferase to platelet ratio index, platelet count to spleen size ratio, and risk score differed significantly between the patients with and those without esophageal varices.
Meaning: These biological parameters can predict esophageal varices among pediatric patients and indicate the need for esophagogastroduodenoscopy.

\section{Introduction}

Liver cirrhosis (LC) is often associated with portal hypertension, which is the major cause of gastroesophageal varices, presented in $40 \%-85 \%$ of the cirrhosis patients. ${ }^{1}$ Visceral bleeding is one of the serious complications associated with cirrhosis and portal vein hypertension, ${ }^{2)}$ with the incidence of 20\%-76\%, ${ }^{3)}$ therefore, early diagnosis is of great importance. Esophagogastroduodenoscopy is a gold-standard diagnostic method for varices, however, invasiveness of the technique and a significant risk associated with sedation on long-term neurological outcomes have limited its use. ${ }^{4)}$ The presence of red color and large varices mark the visceral bleeding in these patients. ${ }^{5)}$ Additionally, other imaging and labbased markers are also utilized to measure liver stiffness, platelet count, and splenomegaly are also studied, yet none of these are adequately efficient for the prediction of the risk.

There is no defined protocol for screening of $\mathrm{EV}$ in pediatrics, to the date. Generally, adult-based recommendations are applied in pediatric patients. Therefore, a noninvasive technique is highly demanded in children. A recent study has highlighted the use of hemostatic markers for predicting upper gastrointestinal bleeding in children with cirrhosis. ${ }^{6}$ Moreover, end-stage liver disease models, aspartate aminotransferase (AST) and alanine aminotransferase (ALT) ratio, AST to platelet ratio index (APRI), platelet count to spleen diameter (PC/SD) have been studied as noninvasive markers for EV in several studies. ${ }^{7)}$ Nonetheless, due to controversies, clinical applications of these markers are still unknown. ${ }^{8)}$

Thus, this study is designed to investigate the role of noninvasive markers for the identification of esophageal varices in children with LC.

\footnotetext{
Corresponding author: Ghobad Heidari, MD. Department of Pediatrics, Faculty of Medicine, Lorestan University of Medical Sciences, Anooshirvan Rezaei Square, Khorramabad, Lorestan 6813833946, Iran 


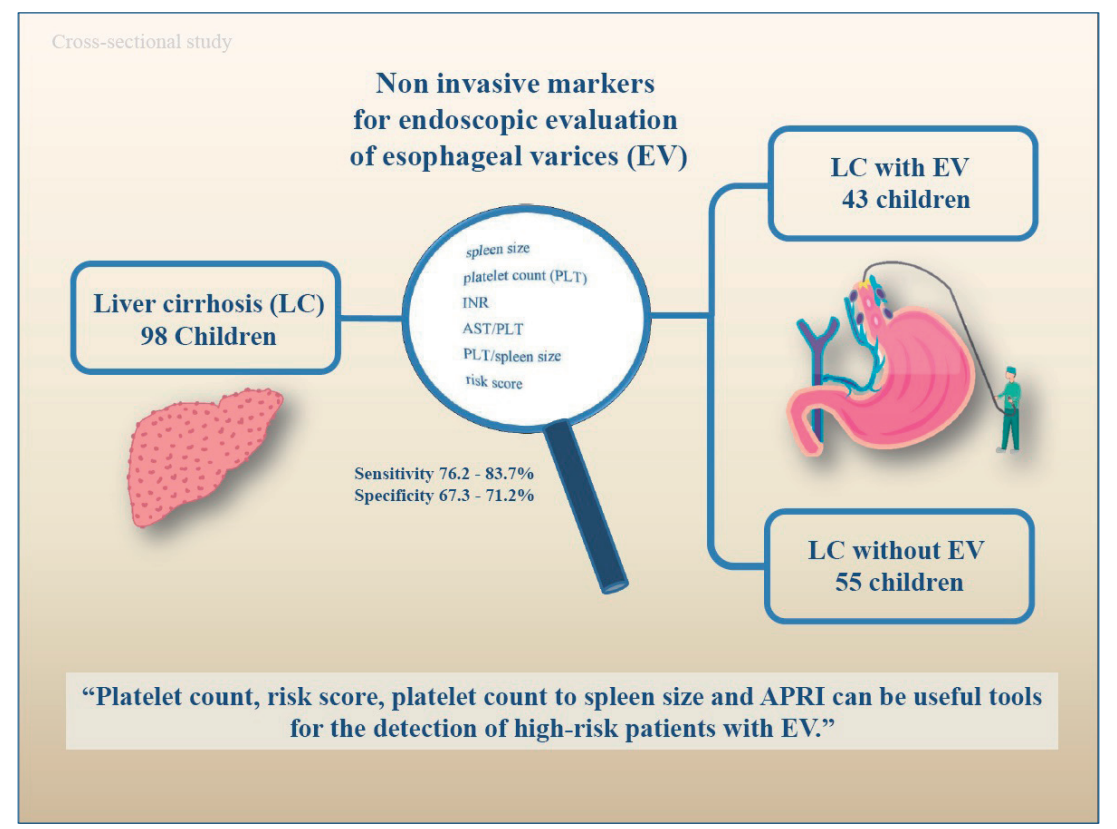

Graphical abstract

\section{Methods}

\section{Study population}

This cross-sectional study included all cirrhotic children who were referred to gastroenterology department of Children's Medical Center, Tehran, Iran. Patients under the age of 18 years who were diagnosed with cirrhosis based on clinical, biochemical, histological (portal hypertensive gastropathy), and ultrasonographic findings were included in this study. ${ }^{9)}$

Exclusion criteria included; active hemorrhage of varicose veins at the time of referral, or history of bleeding, history of treatment for esophageal varicose or history of receiving nonselective beta-blockers or nitrates, ligation or endoscopic sclerotherapy, either surgical shunts or radiologic shunt, transjugular intrahepatic portosystemic shunt or the previous history of liver transplantation or malignancy.

\section{Data collection and clinical analysis}

Clinical data included age, sex, and etiology of the cirrhosis. The presence of splenomegaly, ascites, and hepatic encephalopathy were noted during the physical examination. All patients underwent esophagogastroduodenoscopy (EGD) which was performed by a single hepatologist, and variceal size and ap. pearances (F1, F2, F3), the presence of the red signs, and variceal grading were recorded, as per the provided guidelines by the Japanese Society classification. ${ }^{10)}$ The following noninvasive markers were evaluated for the prediction of the EV: (1) platelet count using automated hematology analyzer (Sysmex XT2000i, Kobe, Japan); (2) spleen diameter; (3) APRI test (AST/ upper limit of normal/platelet count $\left.\left(\times 10^{9} / \mathrm{L}\right) \times 100\right)$; (4) risk score: $\left[14.2-7.1 \times \log 10\right.$ platelets $\left.\left(10^{9} / \mathrm{L}\right)\right]+[4.2 \times \log 10$ bilirubin $(\mathrm{mg} / \mathrm{dL})](1 \rightarrow 20)^{11)}$; measurements were made using enzy. matic method provided by PARS AZMUN (Tehran, Iran) kits by auto analyzer machine (Alcyon 300, Abbott, Abbott Park, IL, USA). Spleen diameter was assessed through ultrasonography by a single-blinded radiologist. Patients fasted 8 hours before ultrasound and patients stayed in supine position with normal breathing during the ultrasound. All tests were blinded and conducted by an experienced sonologist. The observer made 3 consecutive estimation for every variable using Aplio Color Doppler Ultrasound Unit (Toshiba, Tokyo, Japan). The doppler tracing via the lateral intercostal space with a beam angle below 20 was achieved. Portal vein and hepatic artery calculations were conducted by the software provided with the scanner.

A 2-page questionnaire comprising of demographic information, such as age, sex, date of birth, date of onset of symptoms, diagnosis history, family history, clinical and laboratory findings of patients were recorded for each patient. Laboratory examination included: platelet count, spleen size, PC/SD, AST to platelet ratio were investigated. The following data were recorded for the calculation of risk score: the underlying causes of liver disease (including biliary atresia, autoimmune hepatitis, idiopathic biliary cirrhosis, sclerosing cholangitis, cystic fibrosis), laboratory parameters such as complete blood cell, AST, ALT, international normalized ratio (INR), bilirubin, albumin, spleen size based on ultrasound, and the presence or absence of sple. nomegaly, and clinical signs such as encephalopathy.

Ethical approval for the study was obtained from the Tehran University of Medical Sciences Review Board (IR.TUMS. CHMC.REC.1397.037).

\section{Statistical analysis}

Data were presented as the mean and standard deviation, median and interquartile range, and proportions, and $95 \%$ of confidence interval $(\mathrm{CI})$ were reported. A $P$ value of $<0.05$ was 
considered statistically significant. Quantitative variables were analyzed using Student $t$ test or the Mann-Whitney test and qualitative variables by chi-square test or Fisher exact test. With $\mathrm{EV}$ as the dependent variable, variables that were significant on univariate analysis, were evaluated by logistic regression model.

A receiver operator characteristic (ROC) curve was constructed and the area under the ROC curve (AUROC) was calculated. The point of highest sensitivity and specificity was determined as the cutoff value. Sensitivity, specificity, predictive values, and likelihood ratios were calculated for these cutoff values.

\section{Results}

A total of 98 patients with the mean age of $9.48 \pm 4.9$ years were enrolled in the study. Sixty-six patients $(67.3 \%)$ were female and 32 patients (32.7\%) were female. The mean body mass index (BMI) was $17.47 \pm 3.72 \mathrm{~kg} / \mathrm{mm}^{2}$. The etiology of chronic liver disease is summarized in Table 1 . Splenomegaly was seen in 95

Table 1. Patients' demographic details

\begin{tabular}{lc}
\hline Variable & Value \\
\hline Sex, male:female & $66(67.3): 32(32.7)$ \\
Age $(\mathrm{yr})$ & $9.48 \pm 4.90$ \\
Body mass index $\left(\mathrm{kg} / \mathrm{m}^{2}\right)$ & $17.47 \pm 3.72$ \\
\hline
\end{tabular}

Values are presented as number (\%) or mean \pm standard deviation. of the patients (96.7\%) and none of the patients were presented with hepatic encephalopathy. F1, F2, and F3 varices were noted in $13(13.3 \%), 42$ (42.9\%), and 43 patients (43.9\%), respectively. Varices were not detected during EGD in 55 subjects (56.1\%), 43 subjects (43.9\%) presented with varices. The presence of EV did not differ significantly between males and females $(P=0.828)$. Age and etiology of the disease are not different between the groups. Spleen size, platelet count, INR, APRI, platelet count to spleen size ratio, and the risk scores were significantly different in patients with and without varices (Table 2).

On ROC curve analysis, the best predictors of the EV included platelet count, risk score, platelet count to spleen size ratio, and APRI. The AUROC for platelet count, risk score, platelet count to spleen size ratio and APRI were $0.833,0.804,0.794$, and 0.799, respectively (Table 3, Fig. 1). The cutoff points established with the best relationship between sensitivity and specificity for each variable are as follows: platelet count $<111,000$ platelets per $\mu \mathrm{L}$ (sensitivity, 83.7; specificity, 67.3), risk score $>-0.82$ (sensitivity, 83.7; specificity, 70.9), platelet count to spleen size $<6.95$ (sensitivity, 76.6; specificity, 67.3) and APRI >0.45 (sensitivity, 76.2; specificity, 71.2) (Fig. 2).

\section{Discussion}

We investigated simple, reproducible, and routinely available

Table 2. Univariate analysis and logistic regression for esophageal varices

\begin{tabular}{|c|c|c|c|c|}
\hline \multirow{2}{*}{ Variable } & \multirow{2}{*}{ Varices $(n=43)$} & \multirow{2}{*}{ No varices $(n=55)$} & \multicolumn{2}{|c|}{$P$ value } \\
\hline & & & Univariate analysis & Logistic regression \\
\hline $\mathrm{AST}(\mu / \mathrm{L})$ & $70(40-118)$ & $41(26-98)$ & 0.071 & \\
\hline $\operatorname{ALT}(\mu / L)$ & $54(31-81)$ & $38(23-79)$ & 0.126 & \\
\hline $\operatorname{ALP}(\mu / L)$ & $748(570-951)$ & $735(583-1,020)$ & 0.774 & \\
\hline Total bilirubin (mg/dL) & $1.3(1.0-3.7)$ & $1.3(1.0-2.7)$ & 0.446 & \\
\hline Direct bilirubin (mg/dL) & $0.5(0.3-2.5)$ & $0.5(0.3-1.2)$ & 0.667 & \\
\hline INR & $1.3(1.1-1.6)$ & $1.2(1.0-1.3)$ & 0.015 & 0.393 \\
\hline Platelet count (platelets/ $\mu \mathrm{L}$ ) & $54,000(24,000-95,000)$ & $141,000(80,000-235,000)$ & $<0.0001$ & 0.072 \\
\hline Albumin $(\mathrm{g} / \mathrm{dL})$ & $3.7(2.9-4.2)$ & $3.7(3.4-4.1)$ & 0.467 & \\
\hline Spleen size $(\mathrm{cm})$ & $17.2(11.15-19.12)$ & $14.45(9.7-18.9)$ & 0.279 & \\
\hline Platelet count to spleen size ratio $\left(\mathrm{PLT} / \mathrm{cm}^{3}\right)$ & $2.39(1.3-7.28)$ & $10.43(5.7-19.8)$ & $<0.0001$ & 0.296 \\
\hline APRI & $1.28(0.45-3.5)$ & $0.35(0.14-0.69)$ & $<0.0001$ & 0.229 \\
\hline Risk score & $1.67(0.48-3.3)$ & $2.36(3.5-10.43)$ & $<0.0001$ & 0.414 \\
\hline
\end{tabular}

AST, aspartate aminotransferase; ALT, alanine aminotransferase; ALP, alkaline phosphatase; INR, international normalized ratio; PLT, platelets APRI, aspartate; aminotransferase to platelet ratio index.

Boldface indicates a statistically significant difference with $P<0.05$.

Table 3. Area under the receiver operating characteristic curves and 95\% Cls for significant biomarkers

\begin{tabular}{lccccccc}
\hline Variable & Sensitivity & Specificity & PPV & NPV & LR+ & LR- & $95 \%$ Cl \\
\hline Platelet $<111,000 \mathrm{~mm}^{3}$ & 83.7 & 67.3 & 66.7 & 84.1 & 2.50 & 0.24 & $0.754-0.912$ \\
APRI $>0.45$ & 76.6 & 67.3 & 64.7 & 78.7 & 2.34 & 0.30 & $0.704-0.855$ \\
Risk score $>-0.82$ & 83.7 & 70.9 & 69.2 & 84.8 & 2.87 & 0.23 & $0.714-0.894$ \\
Platelet count/spleen size $<6.95$ & 76.2 & 71.2 & 68.1 & 78.7 & 2.64 & 0.33 & $0.714-0.884$
\end{tabular}

$\mathrm{Cl}$, confidence interval; PPV, Positive predictive value; NPV, Negative predictive value; LR+, positive likelihood ratio; LR-, negative likelihood ratio; APRI, aspartate aminotransferase to platelet ratio index. 


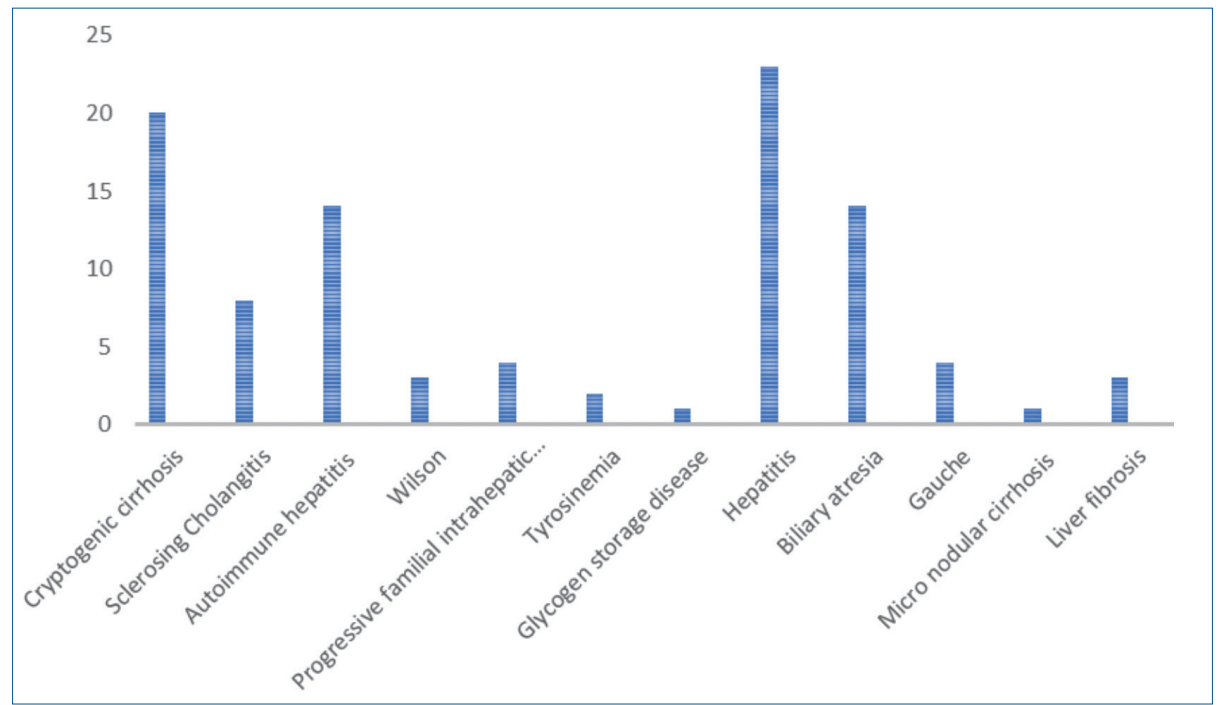

Fig. 1. Causes of liver cirrhosis by frequency.

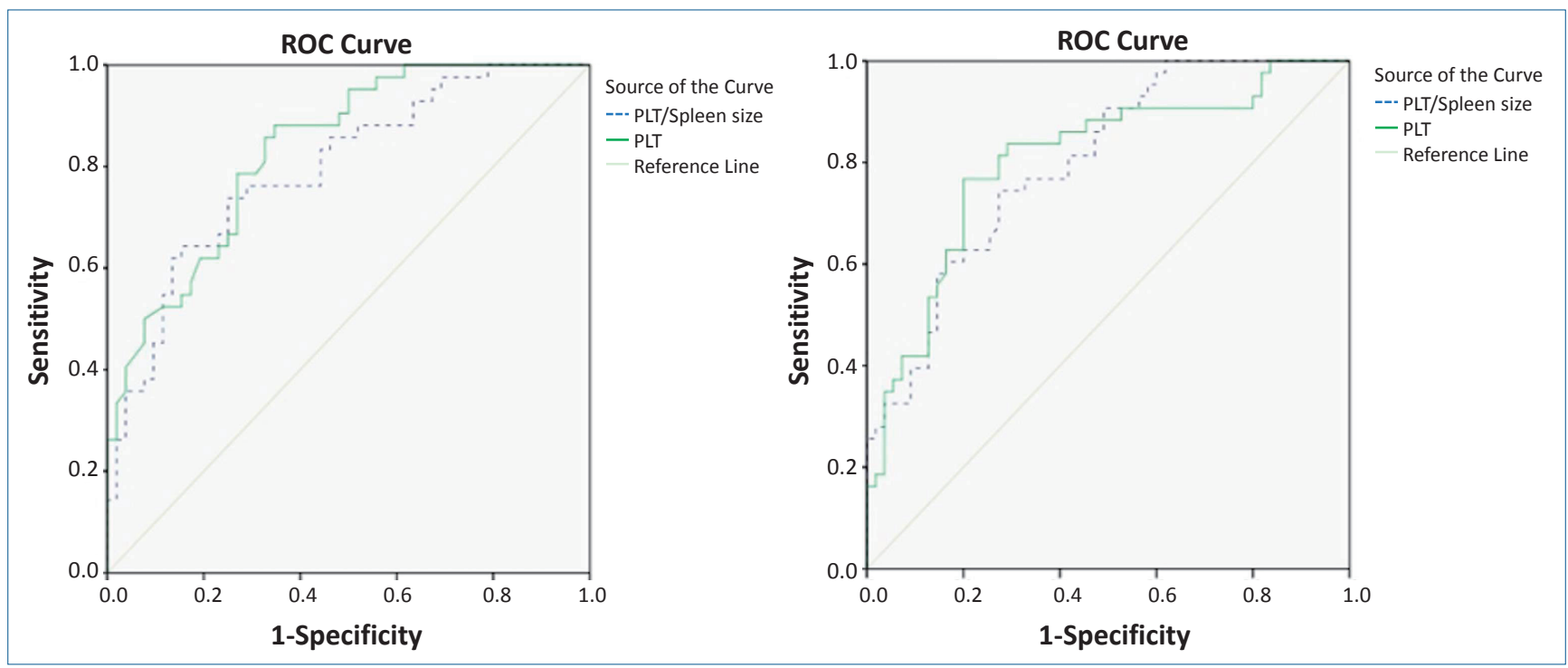

Fig. 2. Receiver operating characteristic (ROC) curves for the presence of esophageal varices and the sensitivity and specificity of the platelet count to spleen size ratio and APRI. APRI, aspartate aminotransferase to platelet ratio index; AST, aspartate aminotransferase; PLT, platelets.

noninvasive parameters for esophageal varies in pediatric LC. Our study indicated platelet count $<111,000$ platelets per microliter, risk score $>-0.82$, platelet count to spleen size $<6.95$, and APRI $>0.45$ can be the significant predictors for the presence of EV. Spleen size, platelet count, INR, APRI, platelet count to spleen size ratio, and the risk score differed significantly between patients with and without $\mathrm{EV}$ on univariate analysis, however, the logistic regression analysis did not show any differences, which also indicates that none of these parameters were independently associated with the presence of the EV. The differences in the age, sex, and the etiology of the disease are possible indicators of these findings. ${ }^{12}$ Several studies have been performed to identify noninvasive markers of $\mathrm{EV}$ in children, such as hypoalbuminemia, the Child-Pugh score, spleen diameter, thrombocytopenia, the platelet to spleen diameter ratio, Clinical Prediction Rule (CPR), and the APRI. ${ }^{13-15)}$ Other modalities, such as computed tomography scan, transient elastography, and endoscopic capsule imaging are also found useful for detecting the large EVs; though they are expensive and are not commonly available. ${ }^{16)}$ Gana et al., ${ }^{17)}$ indicated that platelet count, with a cutoff point of 115,000 , is the best predictor of EV, with an AUROC curve of 0.79 (95\% CI, 0.690-0.900). Our study is in line with their findings, we indicated that the platelet count with a cutoff of 111,000 , can predict the EV, with an AUROC curve of 0.833 (95\% CI, 0.754-0.912). In the adult population with advanced fibrosis, Park et al. ${ }^{11)}$ assessed the laboratory variables for predicting the presence of EV. They combined variables, including the platelets and bilirubin, to form a risk score. They indicated that the score had a good sensitivity and specificity with the cutoff point of -1.0. However, this study was conducted in adults. The current study found that the risk score is a good predictor of EV in children, where, the cutoff point with best sensitivity and specificity was -0.82 (sensitivity, $83.7 \%$; specificity, 70.9\%; odds ratio [OR], 12.54). According to the 
study performed by Adami et al. ${ }^{18)}$ in 2018 on 98 children with portal hypertension, 3 markers, including CPR (OR, 8.59), the risk score (OR, 6.09), and the platelet/spleen size $z$ score below 25 (OR, 3.99) were reported to be good predictors of large EV. The CRP was not investigated in the current study, but our findings were in accord with their findings, in addition to risk score (OR, 12.54) and platelet/spleen size $z$ score (OR, 7.89), we found that the APRI $(\mathrm{OR}, 6.78)$ and platelet count $(\mathrm{OR}$, 10.57) are also good predictors. Fagundes et al. ${ }^{15)}$ evaluated 111 children in 2007 (age range, 0.7-17.6 years) and demonstrated that splenomegaly, Platelet count below the $130,000 / \mathrm{mm}^{3}$ and prehepatic and presinusoidal causes of portal hypertension could predict the presence of EV. Due to a high risk of bleeding, Molleston ${ }^{19)}$ recommended close monitoring of children with portal hypertension associated with splenomegaly and low platelets, which in association with the aforementioned factors with EV, was indicated by Fagundes et al. ${ }^{15)}$ splenomegaly via physical examination has high sensitivity and specificity for the diagnosis of $\mathrm{EV}$. Being an important sign of cirrhosis and portal hypertension, splenomegaly raises the risk of $\mathrm{EV}$ up to 14.62 folds, which was indicated by Fagundes et al. ${ }^{15)}$; as well as hypoalbuminemia which shows portal hypertension and a higher risk of EV (OR, 4.17). Splenomegaly was present in $96.7 \%$ of our patients, but our results did not indicate it as a predictor of $\mathrm{EV}$ in cirrhotic children, moreover, the mean albumin level in our study did not differ between the patients with and without EV.

Thrombocytopenia can occur in these patients as a result of several etiologies, such as immune-mediated mechanisms, lower thrombopoietin synthesis, or platelets pooled by spleen as a result of portal hypertension. ${ }^{20)}$ Without any intermediated factors associated with the pathogenesis, it has been indicated that thrombocytopenia is related directly to portal hypertension, in addition to the presence of $\mathrm{EV}^{21)}$ Unlike adults, ${ }^{22)}$ isolated platelet count could predict EV in pediatrics ${ }^{23}$; however, different cutoff points for platelet count have been described up to present, ranging from $100,000 \mathrm{~mm}^{3}$ to $130,000 \mathrm{~mm}^{3}$. Giannini et al. ${ }^{24)}$ suggested a platelet count to the spleen diameter ratio as a novel predictor of EV. A ratio below the 909 was indicated to be associated with EV where, the diagnostic accuracy of this parameter was $86 \%$ and the negative predictive value was $87 \%$. In this regard, Sezer et al..$^{25)}$ also demonstrated that platelet count and platelet to spleen diameter are unsuitable for detecting the EV in cirrhotic children. Adami et al. ${ }^{14)}$ reported that children with EV had lower platelet count (with cutoff 115,000) and greater spleen diameter. They also found that platelet to spleen ratio below the 1.0 discriminates patients with $\mathrm{EV}$ from those without EV. Although logistic regression was not statistically significant, which was explained by the authors as the differences in the age and gender, we found that it is a significant predictor of $\mathrm{EV}$, with AUROC curve of 0.794 , sensitivity of $76.2 \%$, specificity of $71.2 \%$, positive predictive value of $68.1 \%$, and negative predictive value of $78.7 \%$.

Kim et al. ${ }^{26)}$ suggested APRI as a good predictor of liver fibrosis. Deng et al. ${ }^{27}$ reported that APRI value of 0.608 holds the diagnostic accuracy of EV. Our findings confirmed the accuracy of APRI as a predictor of $\mathrm{EV}$ in children (OR, 6.78; 95\% CI, 2.74-16.75), with the sensitivity of $76.7 \%$ and specificity of $67.3 \%$. According to Sezer et al., ${ }^{25)}$ only the presence of ascites is associated with the presence of $\mathrm{EV}$ in cirrhotic children (age range, 6-18 years). Nonetheless, we failed to confirm these findings in this study.

Our study has some limitations; a larger sample size should be evaluated for identifying the definite noninvasive marker of $\mathrm{EV}$, which could be able to replace the routine endoscopic evaluations performed by experienced hepatologists. We did not divide our patients in age subgroups that might have provided greater specificity to our findings. Furthermore, unlike adults, liver failure in cirrhotic children derives from a variety of causes. It seems better if the accuracy of the markers is evaluated in a group of patients with the same cause of LC.

In conclusion, the findings of our study suggest that platelet count, platelet count to spleen size, and APRI can be the significant predictors for the presence of EV. Additionally, spleen size, platelet count, INR, APRI, platelet count to spleen size ratio, and the risk score differed significantly between patients with and without EV. These factors might reduce the need of invasive methods like EGD.

\section{Conflicts of interest}

No potential conflict of interest relevant to this article was reported.

\section{References}

1. Ma L, Tseng Y, Luo T, Wang J, Lian J, Tan Q, et al. Risk stratification for secondary prophylaxis of gastric varices due to portal hypertension. Dig Liver Dis 2019;51:1678-84.

2. Takehara T, Sakamori R. Remaining challenges for the noninvasive diagnosis of esophageal varices in liver cirrhosis. Esophagus 2020;1:1924.

3. Carneiro de Moura M, Chen S, Kamath BM, Ng VL, Ling SC. Acute variceal bleeding causes significant morbidity. J Pediatr Gastroenterol Nutr 2018;67:371-6.

4. Deng H, Qi X, Guo X. Diagnostic accuracy of APRI, AAR, FIB-4, FI, king, lok, forns, and fibroindex scores in predicting the presence of esophageal varices in liver cirrhosis: a systematic review and meta-analysis. Medicine (Baltimore) 2015;94:e1795.

5. Wang X, Xu Z, Miao CH. Current clinical evidence on the effect of general anesthesia on neurodevelopment in children: an updated systematic review with meta-regression. PLoS One 2014;9:e85760.

6. Bonnet N, Paul J, Helleputte T, Veyckemans F, Pirotte T, Prégardien C, et al. Novel insights into the assessment of risk of upper gastrointestinal bleeding in decompensated cirrhotic children. Pediatr Transplant 2019; 23:e13390.

7. Khadka D, Prajapati S, Sudhamshu KC, Shrestha JK, Karki N, Jaishi B, et al. Significance of non-invasive markers as predictor of esophageal varices in liver cirrhosis. JNMA J Nepal Med Assoc 2017;56:412-6.

8. Kraja B, Mone I, Akshija I, Koçollari A, Prifti S, Burazeri G. Predictors of esophageal varices and first variceal bleeding in liver cirrhosis patients. World J Gastroenterol 2017;23:4806-14.

9. Cherian JV, Deepak N, Ponnusamy RP, Somasundaram A, Jayanthi V. 
Non-invasive predictors of esophageal varices. Saudi J Gastroenterol 2011;17:64-8.

10. Abby Philips C, Sahney A. Oesophageal and gastric varices: historical aspects, classification and grading: everything in one place. Gastroenterol Rep (Oxf) 2016;4:186-95.

11. Park SH, Park TE, Kim YM, Kim SJ, Baik GH, Kim JB, et al. Non-invasive model predicting clinically-significant portal hypertension in patients with advanced fibrosis. J Gastroenterol Hepatol 2009;24:1289-93.

12. Sarangapani A, Shanmugam C, Kalyanasundaram M, Rangachari B, Thangavelu P, Subbarayan JK. Noninvasive prediction of large esophageal varices in chronic liver disease patients. Saudi J Gastroenterol 2010; 16:38-42.

13. Gana JC, Turner D, Roberts EA, Ling SC. Derivation of a clinical prediction rule for the noninvasive diagnosis of varices in children. J Pediatr Gastroenterol Nutr 2010;50:188-93.

14. Adami MR, Ferreira CT, Kieling CO, Hirakata V, Vieira SM. Noninvasive methods for prediction of esophageal varices in pediatric patients with portal hypertension. World J Gastroenterol 2013;19:2053-9.

15. Fagundes ED, Ferreira AR, Roquete ML, Penna FJ, Goulart EM, Figueiredo Filho PP, et al. Clinical and laboratory predictors of esophageal varices in children and adolescents with portal hypertension syndrome. J Pediatr Gastroenterol Nutr 2008;46:178-83.

16. de Franchis R, Primignani M. Natural history of portal hypertension in patients with cirrhosis. Clin Liver Dis 2001;5:645-63.

17. Gana JC, Turner D, Mieli-Vergani G, Davenport M, Miloh T, Avitzur Y, et al. A clinical prediction rule and platelet count predict esophageal varices in children. Gastroenterology 2011;141:2009-16.

18. Adami MR, Kieling CO, Schwengber FP, Hirakata VN, Vieira SMG. Noninvasive methods of predicting large esophageal varices in children with intrahepatic portal hypertension. J Pediatr Gastroenterol Nutr 2018;66:442-6.

19. Molleston JP. Variceal bleeding in children. J Pediatr Gastroenterol Nutr 2003;37:538-45.
20. Giannini E, Botta F, Borro P, Malfatti F, Fumagalli A, Testa E, et al. Relationship between thrombopoietin serum levels and liver function in patients with chronic liver disease related to hepatitis $\mathrm{C}$ virus infection. Am J Gastroenterol 2003;98:2516-20.

21. Shneider BL. Approaches to the management of pediatric portal hypertension: results of an informal survey. In: Groszmann RJ, Bosch J, editors. Portal hypertension in the 21st Century. Dordrecht: Springer, 2004:16772. https://doi.org/10.1007/978-94-007-1042-9_19.

22. Qamar AA, Grace ND, Groszmann RJ, Garcia-Tsao G, Bosch J, Burroughs $\mathrm{AK}$, et al. Platelet count is not a predictor of the presence or development of gastroesophageal varices in cirrhosis. Hepatology 2008;47:153-9.

23. Colecchia A, Di Biase AR, Scaioli E, Predieri B, Iughetti L, Reggiani ML, et al. Non-invasive methods can predict oesophageal varices in patients with biliary atresia after a Kasai procedure. Dig Liver Dis 2011;43:659-63.

24. Giannini E, Botta F, Borro P, Risso D, Romagnoli P, Fasoli A, et al. Platelet count/spleen diameter ratio: proposal and validation of a non-invasive parameter to predict the presence of oesophageal varices in patients with liver cirrhosis. Gut 2003;52:1200-5.

25. Sezer OB, Çelik D, Tutar N, Özçay F. Can platelet count/spleen diameter ratio be used for cirrhotic children to predict esophageal varices? World J Hepatol 2016;8:1466-70.

26. Kim SY, Seok JY, Han SJ, Koh H. Assessment of liver fibrosis and cirrhosis by aspartate aminotransferase-to-platelet ratio index in children with biliary atresia. J Pediatr Gastroenterol Nutr 2010;51:198-202.

27. Deng H, Qi X, Peng Y, Li J, Li H, Zhang Y, et al. Diagnostic accuracy of APRI, AAR, FIB-4, FI, and king scores for diagnosis of esophageal varices in liver cirrhosis: a retrospective study. Med Sci Monit 2015;21:3961-77.

How to cite this article: Rahmani P, Farahmand F, Heidari G, Sayarifard A. Noninvasive markers for esophageal varices in children with cirrhosis. Clin Exp Pediatr 2021;64:31-6. https:// doi.org/10.3345/cep.2019.01599 